Pediat. Res. 3: 19-26 (1969)

Gargoylism liver

keratosulfate Morquio's

Kupffer cells disease

\title{
Etude ultrastructurelle du foie dans la maladie de Morquio
}

\author{
M. Tondeur et H. LoeB ${ }^{[18]}$ \\ Laboratoires d'Anatomie Pathologique et de Microscopie Electronique de l'Université de Bruxelles, \\ et Clinique de Médecine Infantile, Hôpital Universitaire Saint-Pierre, Bruxelles, Belgique
}

\section{Extract}

Liver biopsies from two children with Morquio's disease were studied by electron microscopy. The diagnosis was based upon typical clinical findings and x-ray examination. Urinary excretion of keratosulfate was abnormally high in both cases. The Kupffer cells contained large relatively electronlucid inclusions ( 0.3 to $4.3 \mu$ in diameter) bounded by a single membrane and containing a finely arranged protein-like precipitate. These inclusions occasionally appeared as compact bodies (figs. 2 and 3). Membranous 'myelin-like' round bodies were infrequently seen in the vacuoles. Hepatocytes were usually normal, but some contained a few electron-lucid inclusions that resembled those seen in the Kupffer cells, although they were smaller in size (0.4 to $1.9 \mu$ ) (fig. 5). Peribiliary dense bodies were numerous and rarely contained myelin-like figures (fig.4). In some atypical groups of cells, discontinuous limiting membranes were observed. These cells also contained electron-lucid vacuoles bounded by single membranes of varying sizes $(0.3$ to $4.0 \mu)$ (fig. 6 ).

The inclusions were very similar to those previously described in different forms of gargoylism, although the site was different. The rupture of some Kupffer and/or parenchymal cell membranes, secondary to technical manipulations, apparently caused the peculiar aspect of the atypical groups of cells.

\section{Speculation}

This study supplies new evidence linking Morquio's disease and gargoylism. Some pathogenic mechanisms appear to be similar in these diseases, both of which affect mucopolysaccharides of the liver in a similar way.

\section{Introduction}

A la suite de nombreuses études, parues ces dernières années, concernant les chondrodystrophies liées à une accumulation de mucopolysaccharides, Maroteaux et LAmX [7] ainsi que MaKusick et al. [5] ont pu classer la maladie de Morquio dans le cadre des mucopolysaccharidoses génétiques, au même titre que les diverses formes de gargoylisme.
Dans ce dernier groupe d'affections, l'étude ultrastructurelle du foie a montré des inclusions claires caractéristiques pouvant correspondre à des lysosomes ayant accumulé des mucopolysaccharides [13]. Il n'existe pas, à notre connaissance, de description concernant l'ultrastructure hépatique dans la maladie de de Morquio.

Au cours de ces derniers mois, nous avons eu l'occasion d'observer deux enfants présentant une ostéochon- 
drite déformante. Le diagnostic de maladie de Morquio a été confirmé par la mise en évidence de kératosulfate dans les urines. Des biopsies hépatiques ont pu être pratiquées. Nous rapportons les résultats de leur étude microscopique et ultrastructurelle.

\section{Observations cliniques [17]}

Cas 1

Fillette âgée de 7 1/2 ans, présentant un nanisme du tronc, une dysmorphie faciale, des déformations des membres et des altérations osseuses radiologiques caractéristiques.

Il existe de fines opacités diffuses dans le parenchyme cornéen. L'audiométrie met en évidence une surdité de perception bilatérale. L'intelligence est normale.

L'excrétion urinaire d'acide glucuronique est dans les limites de la normale $(6,7 \mathrm{mg} / \mathrm{l})$. La séparation chromatographique des mucopolysaccharides isole de faibles quantités d'héparitine-sulfate et de chondroïtine-sulfates $\mathrm{A}$ et $\mathrm{B}$ et montre, en outre, la présence d'une fraction correspondant au kératosulfate.

\section{Cas 2}

Garçon âgé de $4 \frac{1}{2}$ ans, présentant également un nanisme avec dysmorphie faciale et déformation des membres et des altérations radiologiques caractéristiques. Les cornées sont normales. Il existe un léger retard du développement mental et le Q.I. est de 75 . Une audiométrie n'a pas pu être pratiquée.

L'excrétion urinaire d'acide glucuronique est de $9,7 \mathrm{mg} / \mathrm{l}$ et on retrouve la fraction correspondant au kératosulfate.

\section{Méthodes}

Les deux biopsies hépatiques ont été prélevées sous anesthésie générale, l'une (cas 1) par une excision triangulaire entre deux points hémostatiques serrés préalablement et l'autre (cas 2), par ponction à l'aiguille.

Pour l'étude en microscopie optique, une partie de la première biopsie a été fixée dans du liquide de Bouin, enrobée à la paraffine et colorée à l'hématoxylineéosine et au trichrome de Masson. La seconde biopsie a également été examinée après coloration au bleu de toluidine de coupes semi-fines pratiquées sur un matériel enrobé en Epon.

Pour l'étude ultrastructurelle, le matériel a été découpé en fragments d'environ $1 \mathrm{~mm}$ de côté, fixé pendant $1 \mathrm{~h} 30$, à $3^{\circ}$, dans du glutaraldéhyde $4,2 \%$ tamponné selon Millonig [8] à $\mathrm{pH} 7,4$. Après un rinçage de 15 heures à $3^{\circ}$ dans le tampon Millonig $0,1 \mathrm{M}$ additionné de $0,54 \mathrm{~g} / 100 \mathrm{ml}$ de glucose, il a été post-fixé 30 minutes au tétroxyde d'osmium $2 \%$ (tampon Mil- lonig sucré $0,1 \mathrm{M}$ pH 7,4). Après déshydratation à l'alcool, les fragments ont été inclus dans de l'épon. Les coupes ultra fines, faites aux microtomes PorterBlum ou LKB, ont été colorées au plomb selon la méthode préconisée par KaRnovsky [3]. Quelques préparations ont été colorées par l'acide phosphotungstique à $10 \%$ en solution aqueuse $(\mathrm{pH} 1,5)$ après oxydation par l'eau oxygénée à $2 \%$ selon la technique décrite par Marinozzr et Gautier [6]. Les coupes, recouvertes d'un film de carbone, ont été examinées au microscope Siemens Elmiscop I ( $80 \mathrm{Kv})$ à des grossissements directs variant de 2500 à 40000 .

\section{Résultats}

\section{Microscopie optique}

Après fixation au liquide de Bouin et inclusion à la paraffine, il n'y a pas de modifications évidentes de l'aspect histologique du parenchyme hépatique (cas 1 et 2). L'examen des coupes semi-fines de la biopsie du

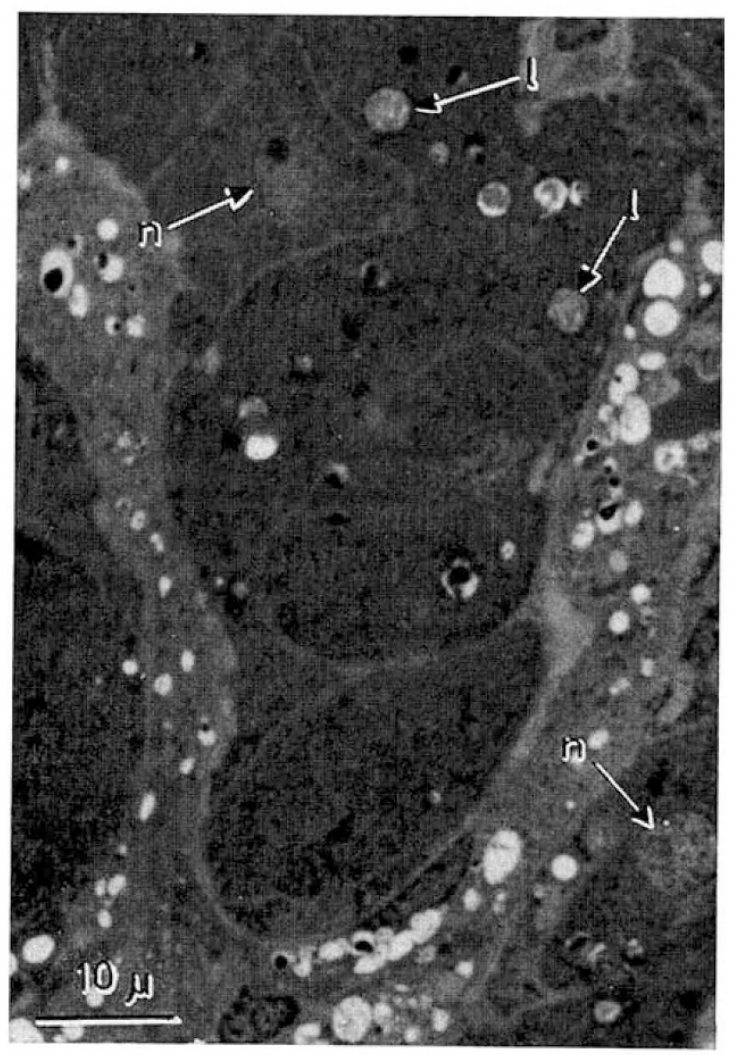

Fig. 1. Case 2. Kupffer cells are filled with numerous vacuoles. Hepatocytes appear normal. n: nuclei; 1: lipid vacuoles. (Fixation: Glutaraldehyde- $\mathrm{OsO}_{4}$; Staining: Toluidine blue). $\times 1600$. 
deuxième cas montre que les cellules de Kupffer sont dilatées par des vacuoles très nombreuses (fig. 1). Les hépatocytes présentent une surcharge lipidique appréciable.

\section{Microscopie électronique}

Des lésions importantes sont visibles au niveau des cellules de Kupffer. Les hépatocytes subissent des altérations beaucoup plus modérées; cependant, certains groupes cellulaires sont tellement altérés par le processus pathologique qu'il est impossible de préciser leur nature exacte. L'espace de Disse présente parfois un certain degré de fibrose.

La cellule de Kupffer. Elle est très dilatée et occupe souvent toute la lumière du sinusoïde. Le noyau est normal. Le cytoplasme fondamental, d'aspect homogène, possède un reticulum endoplasmique pauvre; celui-ci est formé de doubles membranes lisses ou granuleuses et présente par endroits un aspect dilaté vacuolisé. Les mitochondries ont une structure normale. L'appareil de Golgi est abondant et riche en microvésicules. Les particules de glycogène $\beta$ ne sont pas rares.

Le cytoplasme contient un grand nombre d'inclusions claires, plus ou moins sphériques, parfois accolées et communicantes (figs. 2 et 3 ). Elles sont limitées par une membrane unitaire colorable par l'acide phosphotungstique aqueux. Leur diamètre varie de 0,3 à $4,3 \mu$. Un matériel transparent aux électrons constitue l'essentiel de leur contenu. Au sein de celui-ci, des formations plus colorables se différencient:

Une substance modérément osmiophile, dispersée uniformément en flocons ténus (figs. 2 et 3);

Un ou plusieurs nodules denses occupant souvent la partie centrale de l'inclusion (fig. 2) et constitués d'un grand nombre de flocons juxtaposés; dans certains cas, une sphérule homogène plus osmiophile y est accolée;

Des éléments membraneux formant de petites sphérules myéliniques. Ces formations sont rares.

Certaines cellules de Kupffer sont chargées de grosses inclusions de lipides neutres. Leur cytoplasme, riche en ergastoplasme, est comprimé par ces enclaves.

L'hépatocyte. Son noyau est le plus souvent normal; il est rarement chargé de glycogène en particules $\beta$. Les mitochondries et l'appareil de Golgi ont une structure et une abondance habituelles. On observe un petit nombre de microbodies et de corps multivésiculaires; d'assez nombreuses enclaves présentent les caractères morphologiques des lipides neutres.

Les corps denses, nombreux et de grande taille, occupent le plus souvent la zone péribiliaire mais ce n'est pas une règle absolue. Leur constituant principal est un matériel osmiophile granulaire, bien que des amas de lamelles parallèles s'observent également (fig.4); rarement, des petites vacuoles claires sont disséminées dans cette substance dense.
Dans quelques cellules on trouve, en outre, de rares inclusions claires dispersées dans le cytoplasme. Par leur ultrastructure, elles sont semblables à celles des cellules de Kupffer mais leurs dimensions sont sensiblement plus réduites $(0,4$ à $1,9 \mu)$ (fig. 5 ).

Remarquons que l'aspect des hépatocytes n'est pas tout à fait le même dans les 2 cas: dans le premier, les granules de glycogène sont petits et assez rares; le reticulum endoplasmique, modérément dilaté, est rarement granuleux; la plupart des ribosomes sont libres. Dans le deuxième par contre, les particules de glycogène sont grosses et abondantes tandis que le reticulum endoplasmique ne présente aucune altération.

Les amas cellulaires atypiques. A certains endroits, on observe des amas de cellules ballonnées dont les limites sont rendues imprécises par la discontinuité de la membrane plasmatique (fig.6). Un espace extra-cellulaire assez riche en collagène les isole des hépatocytes adjacents. Le cytoplasme fondamental, très homogène, contient un reticulum endoplasmique disloqué. Les mitochondries sont de formes et de dimensions variables. Il n'y a pas d'altération de l'appareil de Golgi. Souvent, des éléments figurés du sang paraissent inclus dans le cytoplasme.

Dans le matériel provenant de la seconde biopsie, ce type de cellule contient de nombreuses particules de glycogène $\alpha$; on n'en trouve pas dans la première biopsie. Dans les deux cas, les vacuoles claires y sont très nombreuses. Leurs dimensions sont variables $(0,3$ à $4,0 \mu)$; leur ultrastructure est identique à celle qui a été décrite à propos des cellules de Kupffer.

Figs.2-6. Fixation: Glutaraldehyde- $\mathrm{OsO}_{4}$; Staining: Karnovsky.

Fig. 2. Case 1. Kupffer cell. The cytoplasm is filled with large clear inclusions in which a fine protein-like precipitate is scattered, sometimes becoming confluent in one or more osmiophilic centers. D: space of Disse; $\mathrm{H}$ : hepatocyte, the plasmatic membrane of which presents numerous microvilli $(\mathrm{mv}) . \times 22200$.

Fig. 3. Case 1. Kupffer cell containing two large clear inclusions with abundant protein-like precipitate. The space of Disse (D) shows large areas of fibrosis (arrows) in which collagen fibers with a periodic ultrastructure are seen (insert: $\times 82000$ ). $\mathrm{m}$ : mitochondria; er: ergastoplasm. $\times 24000$.

Fig. 4. Gase 1. Hepatocyte. Presence of large peribiliary dense bodies containing 'myelin-like' figures. Glycogen $(\mathrm{g})$ is scarce. $\times 24000$.

Fig. 5. Case 2. Hepatocyte. Two small clear inclusions are distinguished. Dense bodies (cd) appear normal. $\mathrm{m}$ : mitochondria; $\mathrm{g}: \alpha$-glycogen. $\times 22200$. 


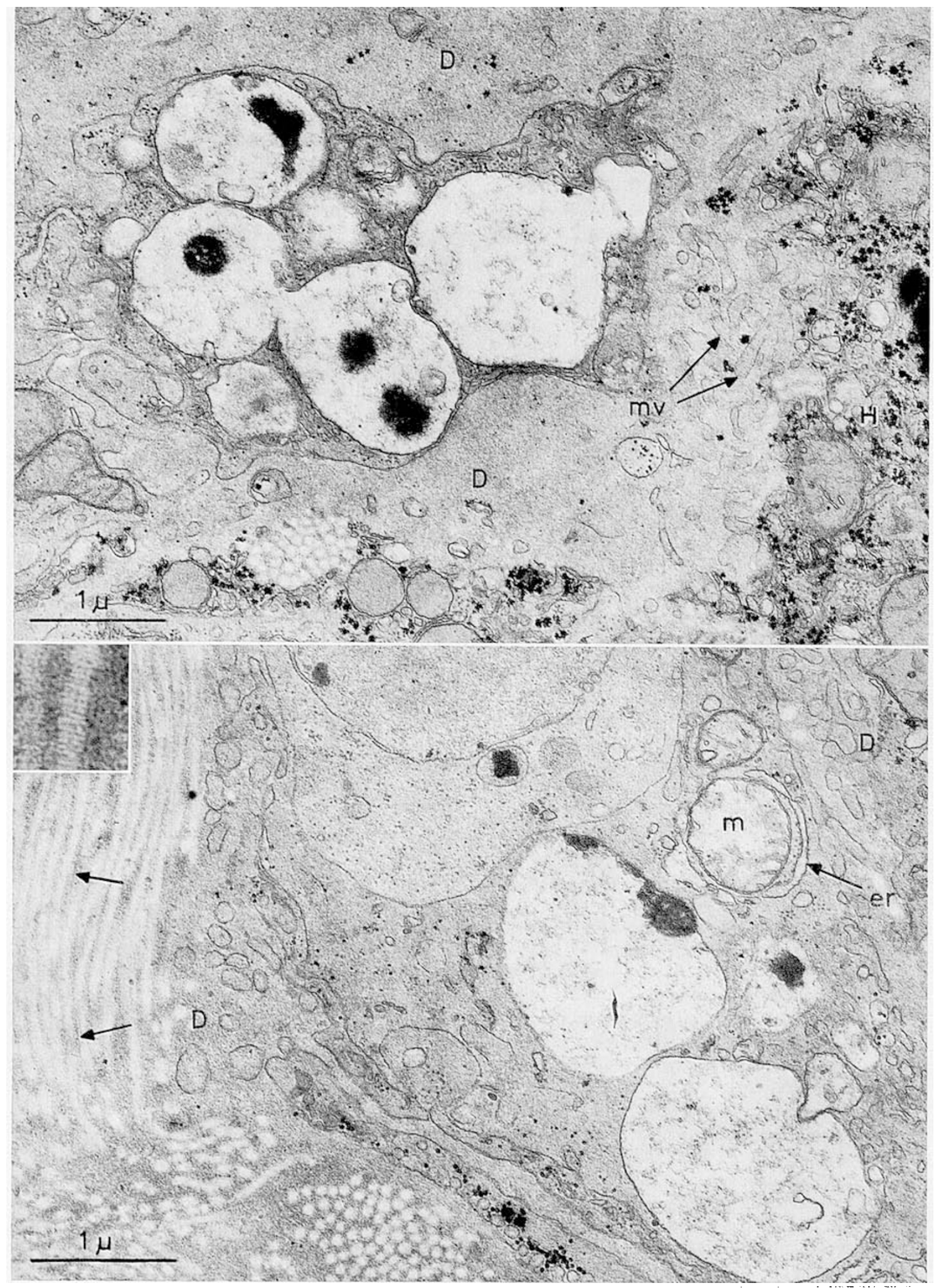




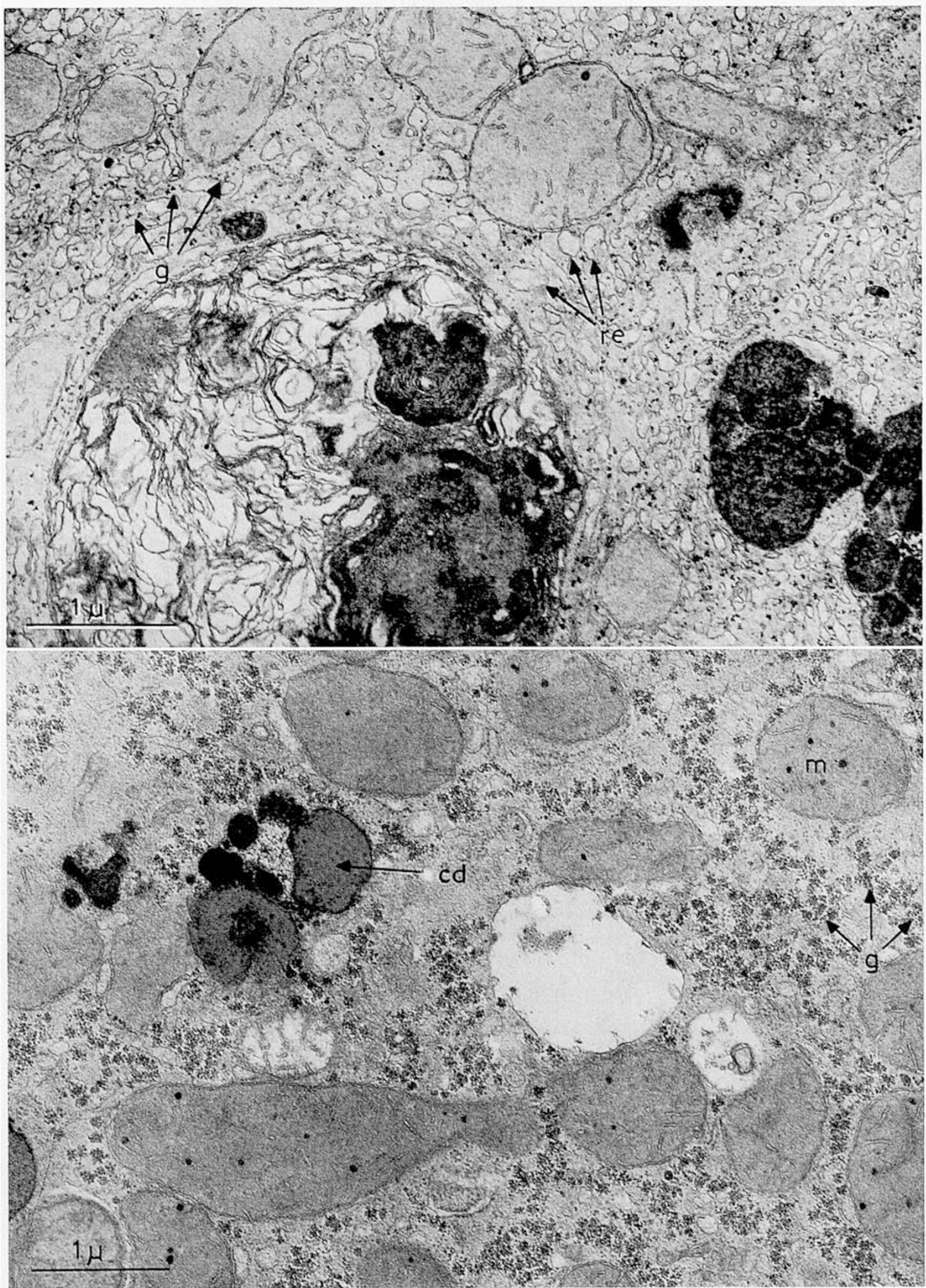


Tondeur, Loeb

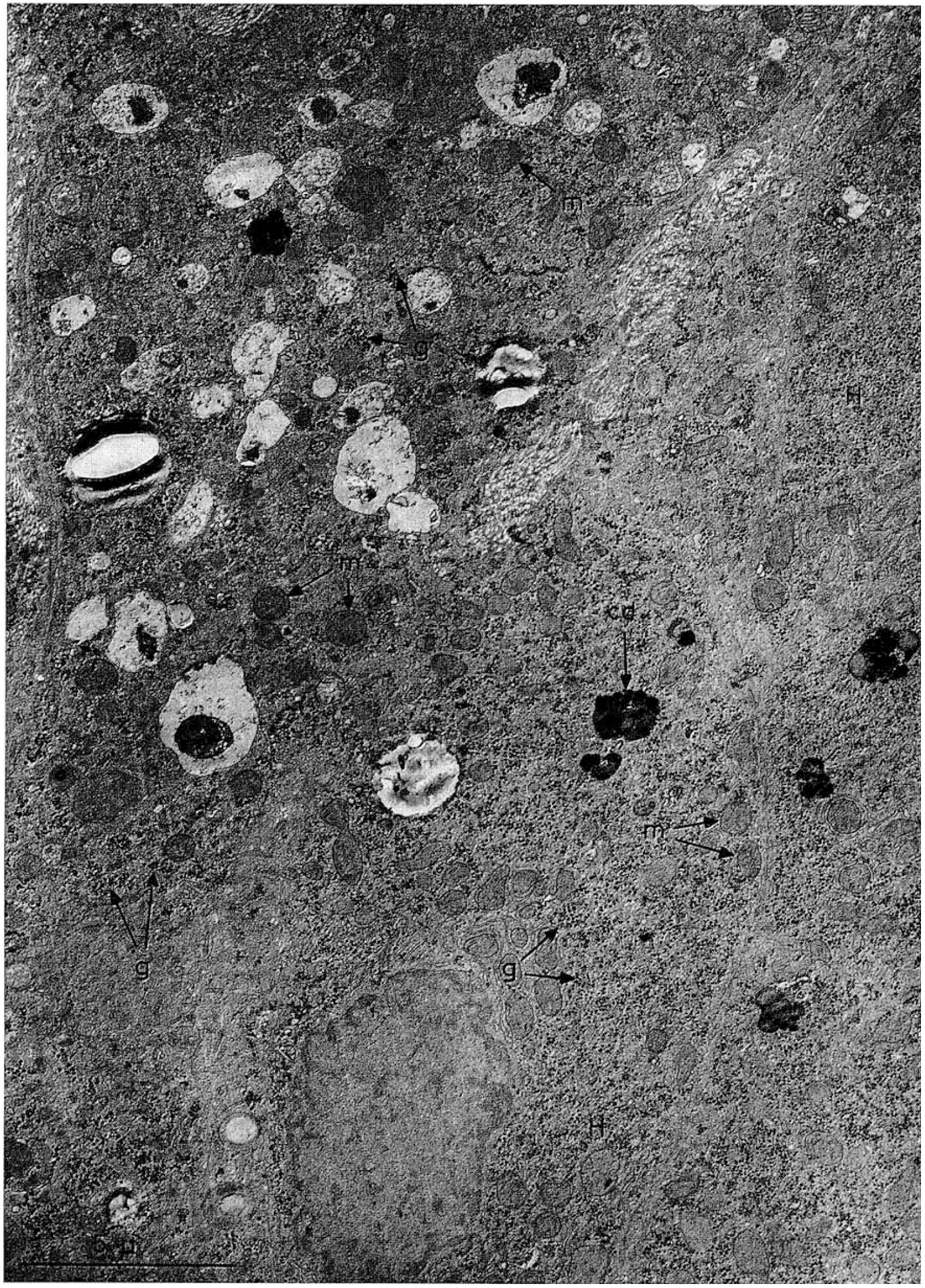


L'espace de Disse. Il est le siège d'une fibrose parfois importante dans le premier prélèvement (fig. 3) et plus discrète dans le second. La membrane bordante de l'hépatocyte présente des microvillosités nombreuses dont l'aspect est normal.

\section{Discussion}

Il n'existe que peu d'études histologiques de la maladie de Morquio. Sahenk et Haggerty [11] ont mis en évidence, par l'étude cyto-chimique, des inclusions mucopolysaccharidiques dans les histiocytes et dans les chondrocytes du cartilage de conjugaison. Par ailleurs; des granulations métachromatiques ont été décrites dans des leucocytes par Zellweger et al. [16].

Différentes variétés de mucopolysaccharidoses (maladies de Hurler-Hunter et de Sanfilippo) ont fait l'objet d'investigations plus complètes. L'étude histologique du foie a montré des inclusions métachromatiques nombreuses de nature mucopolysaccharidique [15]. En microscopie électronique, plusieurs auteurs $[1,2,9,13,14]$ ont décrit des inclusions claires limitées par une membrane simple, dans les hépatocytes et dans les cellules de Kupffer. Ces formations correspondent vraisemblablement aux enclaves métachromatiques. L'aspect vide de leur contenu pourrait s'expliquer par un phénomène de fuite au cours des manœuvres techniques d'un matériau mucopolysaccharidique hautement hydrosoluble [4].

Dans la maladie de Morquio, l'étude ultrastructurelle du foie nous a permis de démontrer que la plupart des cellules de Kupffer sont distendues par de grandes inclusions claires limitées par une membrane unitaire. Ces vacuoles sont également nombreuses dans des amas cellulaires atypiques à limites imprécises dont la nature sera discutée plus loin. Des lésions semblables mais beaucoup plus discrètes, se retrouvent dans un petit nombre d'hépatocytes.

Si l'on compare ces images avec celles qui ont été décrites dans le gargoylisme [13], on constate de grandes similitudes de l'utrastructure des inclusions. Le constituant principal de celles-ci est peut-être également, dans la maladie de Morquio, un mucopolysaccharide hydrosoluble. La surcharge est généralisée dans les maladies de Hurler et de Sanfilippo alors que, dans la maladie de Morquio, ce sont surtout les cellules de Kupffer et les amas cellulaires atypiques qui con-

4 Fig.6. Case 2. Atypical group of cells containing numerous clear inclusions. $\mathrm{cd}$ : dense bodies; $\mathrm{m}$ : mitochondria; $\mathrm{g}: \alpha$-glycogen; $\mathrm{H}$ : hepatocytes. $\times 7200$. tiennent les grandes inclusions claires. Cette distinction, cependant, n'est pas absolue: d'une part, en effet, une localisation exclusivement kupfférienne des lésions a été décrite en microscopie optique, par Wolfe et al. [15], dans un cas de maladie de Hurler; d'autre part, dans la maladie de Morquio, les hépatocytes ne sont pas tout à fait épargnés.

La nature des amas cellulaires atypiques est difficile à interpréter. Il s'agit vraisemblablement d'images pathologiques puisque les descriptions ultrastructurelles du foie normal n'en font pas mention [10]. Par contre, un aspect semblable existe parfois dans la maladie de Hurler (observations personnelles). Il est donc possible que certaines manœurres techniques produisent des ruptures membrannaires au niveau de cellules de Kupffer et (ou) de certains hépatocytes fragilisés par la surcharge. Le cytoplasme coulerait alors dans les espaces voisins entraînant avec lui ses divers organites. Cette hypothèse rendrait compte de l'aspect imprécis des limites cellulaires, de l'homogénéité du cytoplasme dont le reticulum paraît disloqué, ainsi que de la présence de glycogène dans une des biopsies.

L'aspect dégranulé et vacuolisé du reticulum endoplasmique, ainsi que la pauvreté en glycogène des hépatocytes décrits dans la première biopsie sont probablement des altérations accessoires. Des images semblables ont en effet été décrites chez l'animal par STEINER [12] au début de l'ischémie hépatique expérimentale. Dans notre:cas, un phénomène semblable se serait produit au moment du prélèvement chirurgical entre deux points hémostatiques.

\section{Résumé}

L'étude ultrastructurelle de biopsies hépatiques effectuées chez deux enfants atteints de maladie de Morquio a permis de mettre en évidence de grandes inclusions claires localisées dans le cytoplasme de différents types cellulaires.

Ces altérations sont particulièrement intenses dans les cellules de Kupffer, beaucoup plus modérées dans les hépatocytes, et importantes dans des amas cellulaires atypiques dont la nature est difficile a déterminer.

La similitude de ces lésions avec celles qui ont déjà été décrites dans d'autres mucopolysaccharidoses est soulignée.

\section{Références et Notes}

1. Gallahan, W.P. and Lorincz, A.E.: Hepatic ultrastructure in the Hurler syndrome. Amer.J. Path. $48: 277$ (1966).

2. Dodion, J.; Resibors, A.; Loeb, H. et Cremer, N.: 
Etude d'un cas d'oligophrénie polydystrophique (mucopolysaccharidose H. S.). Path. europ. 1: 50 (1966).

3. Karnovskiy, M.J.: Simple methods for 'staining with lead' at high $\mathrm{pH}$ in electron microscopy. J. cell Biol. 11: 729 (1961).

4. Lagunoff, D. and Gritzka, T.L.: The site of mucopolysaccharide accumulation in Hurler's syndrome. An electron microscopic and histochemical study. Lab. Invest. 15: 1578 (1966).

5. McKusick, V. A.; Kaplan, D.; Wise, D.; Hanley, W.B.; Suddarth, S. B.; Sevick, M.E. and MauMANEE, A.E.: The genetic mucopolysaccharidoses. Medicine 44: 445 (1965).

6. Marinozzi, V. et Gautier, A.: Essais de cytochimie ultrastructurelle. Le rôle de l'osmium réduit dans les «colorations» électroniques. C.R.Acad. Sci. (Paris) 253: 1180 (1961).

7. Maroteaux, P. et Lamy, M.: La maladie de Morquio. Etude clinique, radiologique et biologique. Presse méd. 71: 2091 (1963).

8. Mrllonig, G.: Further observations on a phosphate buffer for osmium solutions. Transactions V. int. Conf. Electron Microscopy, Philadelphia, p. 8 (Academic Press, New York/London 1962).

9. Orizaga, M.; Haust, M.D.; Bryans, A.M. and FRANK, H.: Electron microscopic study of liver in Hurler's syndrome. Fed. Proc. 22: 484 (1963).

10. Rouiller, C. and JeZequel, A.M. : Electron microscopy of the liver: in The liver (ed. Rouiller, C.), p. 195 (Academic Press, New York/London 1963).
11. Schenk, E.A. and Haggerty, J.L.: Morquio's disease. A radiologic and morphologic study. Pediatrics 34: 839 (1964).

12. Steiner, J.W.: Ultrastructural and subcellular pathology of the liver. Int. Rev. exp. Path. 3: 65 (1964).

13. VAN Hoof, F. et Hers, H. G.: L'ultrastructure des cellules hépatiques dans la maladie de Hurler (Gargoylisme). C.R. Acad.Sci. Paris 259: 1281 (1964).

14. Wallace, B.J.; Kaplan, D.; ADachi, M.; SchNeGK, L. and Volk, B.W.: Mucopolysaccharidosis type III. Morphologic and biochemical studies of two siblings with Sanfilippo syndrome. Arch. Path. 82: 462 (1966).

15. Wolfe, H.J.; Blennerhasset, J. B.; Young, G.F. and Cohen, R.B.: Hurler's syndrome: A histochemical study. New techniques for localization of very water-soluble acid mucopolysaccharides. Amer.J. Path. 45: 1007 (1964).

16. Zellweger, H.; Ponsetr, I.V.; Pedrini, V.; Stamler, F.S. and Von Noorden, G.K.: Morquio-Ullrich's disease. J. Pediat. 59: 459 (1961).

17. Les données cliniques, radiologiques et biologiques seront detaillées par ailleurs (Dodion et al.: Acta paediat. belg., sous presse).

18. Requests for reprints should be addressed to: H.Loeb, M.D., Clinique de Médecine Infantile, Hôpital Universitaire Saint-Pierre, Rue Haute, 320, Bruxelles (Belgique). 